\title{
Choroidal Blood Flow After Intravitreal Ranibizumab in Vitrectomized and Non-Vitrectomized Eyes with Diabetic Macular Edema
}

\author{
Bernardete Pessoa (1D ${ }^{1,2, *}$ \\ João Heitor Marques (1D ${ }^{1, *}$ \\ João Leite' \\ Nisa Silva $\mathbb{D}^{\prime}$ \\ Diana José (iD) \\ Constança Coelho (iD ${ }^{3}$ \\ João Figueira $\mathbb{1}^{4-6}$ \\ Angelina Meireles (D) ${ }^{1,2}$ \\ João Nuno Melo-Beirão ${ }^{1,2}$ \\ 'Departamento de Oftalmologia, \\ Hospital de Santo António, Centro \\ Hospitalar Universitário do Porto, Porto, \\ Portugal; ${ }^{2}$ Instituto de Ciências \\ Biomédicas Abel Salazar (ICBAS), \\ Universidade do Porto, Porto, Portugal; \\ ${ }^{3}$ Instituto de Saúde Ambiental, Faculdade \\ de Medicina, Universidade de Lisboa, \\ Lisboa, Portugal; ${ }^{4}$ Centro Hospitalar e \\ Universitário de Coimbra, Coimbra, \\ Portugal; ${ }^{5}$ Faculty of Medicine, University \\ of Coimbra, Coimbra, Portugal; \\ ${ }^{6}$ Association for Innovation and \\ Biomedical Research on Light and Image, \\ Coimbra, Portugal \\ *These authors contributed equally to \\ this work
}

Correspondence: Bernardete Pessoa Email bbtpessoa@gmail.com

\begin{abstract}
Aim: Diabetic retinopathy staging system and progression predictors are soon to be considered insufficient for ophthalmologic practice. Given the growing evidence of the role of choroidal dysfunction, our purpose was to assess choroidal vascular changes with intravitreal ranibizumab (RBZ) treatment in diabetic macular edema (DME).
\end{abstract}

Methods: This was a prospective longitudinal cohort study. The study included DME eyes, grouped in vitrectomized (group 1) and non-vitrectomized (group 2) eyes, submitted to RBZ in a pro re nata regimen, with 24 weeks of follow-up. Main outcome measures such as central subfield foveal thickness (CFT), choroidal thickness (CT), and choroidal vascular index (CVI) were obtained from structural OCT, and choriocapillaris flow density (CCD) was obtained from OCT angiography and analyzed before and after treatment.

Results: Thirty-one patients were included, 10 eyes in group 1 and 24 eyes in group 2. The mean number of injections was 5.18 (range 2-6). Globally, there was an improvement in BCVA (+4.3 ETDRS letters, $\mathrm{p}=0.004)$ and CFT $(-84.6 \mu \mathrm{m}, \mathrm{p}<0.001)$ with no changes in CT, CVI, or CCD ( $p>0.05)$. When considering only group 2 , there was a significant decrease in $\mathrm{CT}(\mathrm{p}=0.033)$ and a significant increase in $\mathrm{CCD}(\mathrm{p}=0.010) 6$ months after treatment, with no differences in CVI $(\mathrm{p}=0.111)$. Baseline CVI was correlated with visual acuity at week 24 both globally $(r=0.406, \mathrm{p}=0.029)$ and in group $2(r=0.604, \mathrm{p}=0.004)$.

Conclusion: In non-vitrectomized eyes, choriocapillaris blood flow improves with RBZ. Baseline CVI may correlate with visual function after RBZ. ClinicalTrials.gov NCT04387604.

Keywords: diabetic macular edema, ranibizumab, vitrectomy, choroidal vascular index, choriocapillaris flow

\section{Introduction}

Diabetic retinopathy is the leading cause of preventable blindness among workingage individuals in most developed countries, and diabetic macular edema (DME) is the main cause of visual impairment in diabetic patients. DME pathogenesis is complex, multifactorial, and not completely understood. Several mechanisms such as oxidative damage, microvascular hypoperfusion, and up-regulation of vascular endothelial growth factor (VEGF) are implicated in increased fluid leakage into retinal tissue. ${ }^{1,2}$ Clinical and experimental findings suggest that not only the retinal capillaries but also choroidal vasculopathy in diabetes play a role in the pathogenesis of diabetic retinopathy. ${ }^{3-5}$ Obstruction of the choriocapillaris, vascular degeneration, choroidal aneurysms, and choroidal neovascularization have been reported 
in histopathologic studies of diabetic eyes. ${ }^{3}$ Central choroid thinning in type 2 diabetic eyes and choroidal blood flow reduction in eyes with DME have already been described. $^{5,6}$

There is some controversy regarding the real impact of anti-VEGF therapy in choroidal and retinal vessels. VEGF has an undoubtful role in normal vascular physiology through neurovascular trophic effects, namely on endothelial survival and in the allowance of some fenestration of vascular tissue in adults. ${ }^{7}$ In the choroid, VEGF receptors have been identified both in the choriocapillaris and in large blood vessels. ${ }^{8,9}$ Additionally, it has been shown that the choroid is highly dependent on VEGF. ${ }^{9}$

Although there is evidence that sustains that antiVEGF therapy slows or even reverses retinal nonperfusion in diabetic retinopathy, ${ }^{10}$ Falavarjani et al demonstrated that there are no differences in the retinal blood flow before and after a single intravitreal injection (IV) of anti-VEGF for macular edema. ${ }^{11}$ In choroidal tissue, Okamoto et al observed that a single anti-VEGF IV reduces choroidal vascular index (CVI) and choroidal blood flow in DME eyes not previously submitted to panretinal photocoagulation (PRP). ${ }^{12}$ The long-term effect of anti-VEGF intravitreal therapy in choroidal vasculature is much less explored.

Our purpose was to analyze choroidal vascular changes with intravitreal ranibizumab (RBZ) treatment in a pro re nata (PRN) regimen in DME during 24 weeks of follow-up.

\section{Materials and Methods Study Design}

This was a single-center, prospective, observational study, conducted at the Department of Ophthalmology of Centro Hospitalar e Universitário do Porto (CHUP), Portugal. This study was conducted according to the tenets of the Declaration of Helsinki in its latest amendment (Brazil, 2013) and was approved by the ethics committee of CHUP [2017.093 (084-DEFI/082-CES)]. All patients signed an informed consent form. This study is registered at www. clinicaltrials.gov (NCT04387604).

\section{Study Population}

The recruitment period was from January 2018 to January 2019. Patients were included in two groups according to the vitreous status: group 1 - vitrectomized eyes and group 2 - non-vitrectomized eyes. Inclusion criteria were as follows: 1 ) $\geq 18$ years with either type 1 or type 2 diabetes mellitus; 2) central subfield foveal thickness (CFT) $>300 \mu \mathrm{m}$ and a minimum signal strength for optical coherence tomography angiography of 7 out of 10, measured using spectral domain optical coherence tomography (SD-OCT); 3) best-corrected visual acuity (BCVA) of 20 to 80 letters, using Early Treatment of Diabetic Retinopathy Study (ETDRS) letters chart; 4) minimum period of 6 months post-vitrectomy for inclusion in group 1 ; 5) ability and willingness to provide written informed consent. If the inclusion criteria were fulfilled for both eyes, bilateral inclusion was allowed. Exclusion criteria were as follows: 1) epiretinal membrane (ERM) in the study eye; 2) persistent posterior hyaloid adherence after vitrectomy for group $1 ; 3$ ) history of other retinal vascular diseases in the study eye; 4) scatter and macular LASER photocoagulation, IV anti-VEGF, systemic antiVEGF, pro-anti-VEGF treatment or cataract surgery in the 6 months prior to study inclusion; 5) IV or peribulbar corticosteroid injections in the 6 months prior to study inclusion; 6) previous IV implant of fluocinolone acetonide in the study eye; 7) vitreous hemorrhage or opacification in the study eye; 8) active proliferative diabetic retinopathy (PDR) in the study eye; 9) active ocular inflammation or infection in either eye; 10) aphakia in the study eye; 11) other causes for macular edema, eg, after cataract surgery in the study eye; 12) other causes of visual loss in the study eye; 13) pro-edematous medication (such as systemic glitazones or topical prostaglandins) or other pathologies that might influence the course of macular edema in the study eye; 14) uncontrolled glaucoma in either eye (intraocular pressure $>24 \mathrm{mmHg}$ with treatment); 15) pathologic myopia (spherical equivalent of $\geq-8$ diopters, or axial length of $\geq 25 \mathrm{~mm}^{19}$ ); 16 ) history of stroke in the previous 6 months; 17) uncontrolled arterial hypertension (systolic blood pressure $>160 \mathrm{mmHg}$ or diastolic blood pressure $>100 \mathrm{mmHg}$ ).

\section{Study Protocol}

Patients were followed according to the standard of care, with visits every 4 weeks. All patients were treated with RBZ IV injections $(0.5 \mathrm{mg} / 0.05 \mathrm{~mL})$ following a PRN regimen. No adjunct therapies such as LASER were admitted during the 24 weeks of follow-up.

\section{Treatment Schedule}

Injections were repeated every 4 weeks if eye "improved" or "worsened" (defined as $\geq 5$ letter change from the last injection, or $\geq 10 \%$ CFT increase on OCT from the last 
injection), or if $\mathrm{CFT}>300 \mu \mathrm{m}$ at any time point. Injections were deferred if either BCVA of 85 letters and OCT CFT was "normal" (CFT $\leq 300 \mu \mathrm{m}$ and non-existent intra- or subretinal fluid); or OCT CFT was "normal" $(\mathrm{CFT} \leq 300 \mu \mathrm{m})$ and stable BCVA (defined as $<5$ letters change from the last injection) after two consecutive injections during the first 24 weeks.

\section{Patient Assessment}

At baseline, demographic and clinical data, including serum levels of hemoglobin and glycated hemoglobin (HbA1C), microproteinuria, body mass index (BMI), and systolic and diastolic blood pressure (BP), were recorded. Each patient underwent a complete ophthalmological evaluation (ocular symptoms, BCVA, intraocular pressure measurement, anterior and posterior segment biomicroscopy), OCT and OCT-angiography (OCT-A) exams at baseline and every 4 weeks until the end of follow-up. BMI and BP were assessed at every visit. Fluorescein angiography was performed on all patients at baseline and at the end of follow-up. A final visit was conducted at 24 weeks of follow-up, which was the minimum followup period required for each patient.

\section{OCT Acquisition and Imaging Protocol}

At every visit, two highly trained technicians conducted a fovea-centered $20^{\circ} \times 20^{\circ}$ SD-OCT scan (Spectralis ${ }^{\circledR}$ OCT, version 1.10.2.0, Heidelberg Engineering, Heidelberg, Germany) to automatically assess central foveal thickness (CFT) in the central 1-millimeter circle, using the proprietary Heidelberg Eye Explorer ${ }^{\circledR}$ software. Choroidal imaging was performed using structural OCT and OCT-angiography (OCT-A). CVI was calculated in a single $3 \mathrm{~mm}$-cropped image exported from a structural high-resolution foveal-centered B-scan. In

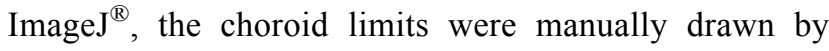
two experienced examiners (DJ, SP) that were masked to patient clinical data at the time of acquisition. Segmentations were repeated by a senior specialist for all cases where differences between the two masked observers were equal or superior to $20 \%$. The senior specialist used the same method described above and was also masked to previous measurements. The outer border of the hyperreflective line representing the retinal pigment epithelium was considered the inner choroidal limit. The outer choroidal limit was defined as the inner border of the hyperreflective sclera. The presence of a visible suprachoroidal space (SCS), defined as a continuous homogenous hyporeflective layer between the choroid and the sclera, was recorded and, in such cases, SCS was not considered part of the choroid. As previously described by other groups, ${ }^{39}$ the image was binarized using the Niblack method, and the total choroidal area (CA), hyperreflective stromal area, and hyporeflective vascular luminal areas (LA) were automatically calculated. CVI was calculated as LA/CA. CA was converted to average choroidal thickness (CT). Choriocapillaris flow density (CCD) was calculated from a $10^{\circ} \times 10^{\circ}$ OCT-A C-scan, segmented automatically in the choriocapillaris layer using the OCT proprietary software. The image was binarized in Image $J^{\circledR}$ using Phansalkar's method ${ }^{40}$ and CCD was defined as the ratio between vascular area and total area. Auto-rescan with active eye tracking was applied for OCT acquisitions. The minimum signal strength of OCT-A images was 7 of 10. The signal strength index was registered and compared between visits, given that it may directly influence the outcomes. Automatic segmentations were confirmed by the investigators (BP, JH). OCT was performed at the same time of the day, between 8 a.m. and 10 a.m, to avoid circadian fluctuations. ${ }^{18}$

\section{Outcome Measures}

\section{Primary Outcome}

To analyze choroidal vascularity changes, through CVI, CCD, and CT, 6 months after PRN treatment with RBZ.

\section{Secondary Outcomes}

Secondary endpoints were as follows: 1) BCVA and CFT 6 months after treatment; 2) prognostic value of baseline choroidal measurements for functional (BCVA) and anatomical (CFT) response at 12 weeks (early response) or 24 weeks of follow-up; 3 ) correlation between choroidal vascularity parameters at baseline and after 6 months; 4) effect of baseline and demographic characteristics in choroidal vascularity parameters at baseline and after treatment; 5) BP and BMI association with choroidal vascularity parameters, at baseline and at the end of follow-up.

\section{Functional and Anatomical Response Criteria}

Functional and anatomical improvements were defined as a gain of $\geq 5$ ETDRS and a CFT decrease of $\geq 50 \mu \mathrm{m}$, respectively. DME resolution was defined as a CFT $\leq 300 \mu \mathrm{m}$. 


\section{Statistical Analysis}

Data were analyzed using non-parametric statistics. Between-group analyses of continuous variables were performed using the Mann-Whitney $U$-test. Within-group analyses were performed using the Wilcoxon test. Nominal variables were analyzed using the $\chi^{2}$ test. Correlations between variables were tested using the Spearman rank correlation or Kendall's $\tau$-b, as appropriate. A $p<0.05$ (two-sided) was considered statistically significant.

\section{Results}

Fifty consecutive eyes with DME were considered for treatment with RBZ IV injections, of which 34 eyes (10 vitrectomized and 24 non-vitrectomized) from 31 patients fulfilled the inclusion criteria and completed the 24-week follow-up period.

\section{Demographic and Clinical Baseline Data}

Comparison between groups regarding baseline demographic and clinical characteristics are summarized in Table 1. The only difference observed between groups was DM duration, which was longer in group 1 $(p=0.020)$. Causes for vitrectomy were PDR in seven cases and ERM in one case. In these eight cases the internal limiting membrane was peeled after membrane blue staining. One vitreous hemorrhage with no PDR and one macula on retinal detachment were registered.

\section{Associations with Choroidal Vascularity at Baseline}

Regarding the choroidal vascularity parameters, CVI, CCD, and CT, there were no baseline differences between groups $(p>0.05)$. There was no association between baseline CVI, CCD, and CT and previous treatment with intravitreal injections, macular laser, or pan-retinal photocoagulation $(\mathrm{p}>0.05)$. At baseline, age and BP were not associated with baseline CT, CVI, or CCD.

\section{Number of Injections in the 24-Week Period}

The mean number of injections during the study period was $5.18 \pm 1.17$ (minimum 2 and maximum 6), with no significant differences between groups (5.70 \pm 0.48 in group 1 and $4.96 \pm 1.30$ in group $2, p=0.080$ ). Treatment burden was moderately correlated with years on insulin

Table I Baseline Characteristics

\begin{tabular}{|c|c|c|c|}
\hline Parameter & Group I $(n=10)$ & Group $2(n=24)$ & p-value \\
\hline Age, mean $[95 \% \mathrm{Cl}]$ & $66.5[60.2-72.8]$ & $68.4[65.1-7 \mid .7]$ & 0.467 \\
\hline Gender female, $\mathrm{n}(\%)$ & $5(50.0)$ & $14(58.3)$ & 0.718 \\
\hline DM duration (years), mean $[95 \% \mathrm{Cl}]$ & $22.1[16.5-27.7]$ & $15.7[12.4-19.0]$ & 0.020 \\
\hline DME duration (months), mean $[95 \% \mathrm{Cl}]$ & $34.0[7.8-60.2]$ & $23.0[13.7-32.4]$ & 0.619 \\
\hline Type 2 DM, n (\%) & $10(100.0)$ & $24(100.0)$ & 1.000 \\
\hline Macular LASER, n (\%) & $8(80.0)$ & $15(62.5)$ & 0.437 \\
\hline PRP LASER, n (\%) & $8(80.0)$ & $13(54.2)$ & 0.251 \\
\hline Naive IV, n (\%) & $2(20.0)$ & $6(25.0)$ & 1.000 \\
\hline $\mathrm{HbAlc}$, mean $[95 \% \mathrm{Cl}]$ & $7.6[6.8-8.3]$ & $7.3[6.9-7.7]$ & 0.589 \\
\hline Hemoglobin, mean $[95 \% \mathrm{Cl}]$ & $\mid 3.1[\mid 1.8-14.4]$ & $13.1[12.3-13.9]$ & 0.985 \\
\hline Microproteinuria, mean $[95 \% \mathrm{Cl}]$ & II 2.7 [19.8-245.3] & $|8| . \mid[5 \mid .7-310.6]$ & 0.908 \\
\hline BMI, mean $[95 \% \mathrm{Cl}]$ & $28.0[25.1-30.9]$ & 27.8 [26.3-29.3] & 0.724 \\
\hline SBP, mean $[95 \% \mathrm{Cl}]$ & $138.5[127.1-149.9]$ & $\mid 38.4[133 .|-| 43.7]$ & 0.832 \\
\hline DBP, mean $[95 \% \mathrm{Cl}]$ & 74.3 [65.6-83.0] & 77.1 [73.6-80.6] & 0.406 \\
\hline BCVA (letters), mean [95\% Cl] & $63.3[52.5-74.7]$ & $72.3[52.5-74.7]$ & 0.196 \\
\hline CFT $(\mu \mathrm{m})$, mean $[95 \% \mathrm{Cl}]$ & $400.5[343.1-457.9]$ & 433.7 [390.7-476.7] & 0.381 \\
\hline CVI, mean $[95 \% \mathrm{Cl}]$ & $0.633[0.603-0.662]$ & $0.64 \mid[0.6 I|-0.67|]$ & 0.324 \\
\hline CCD (\%), mean $[95 \% \mathrm{Cl}]$ & $0.392[0.337-0.448]$ & $0.373[0.319-0.428]$ & 0.764 \\
\hline $\mathrm{CT}$, mean $[95 \% \mathrm{Cl}]$ & $233.7[172.1-295.3]$ & $256.1[232.1-280.1]$ & 0.349 \\
\hline
\end{tabular}

Note: Significant P-value in bold.

Abbreviations: Group I, vitrectomized eyes; Group 2, non-vitrectomized eyes; 95\% CI, 95\% confidence interval; DM, diabetes mellitus; DME, diabetic macular edema; macular LASER, prior focal-grid photocoagulation treatment in the study eye; PRP LASER, Prior panretinal photocoagulation treatment in the study eye; IV, intravitreal; naïve IV, no prior anti-VEGF intravitreal treatment in study eye; CCT, corticosteroid; BMI, body mass index; SBP, systolic blood pressure; DBP, diastolic blood pressure; CVI, choroidal vascularity index; CCD, choriocapillaris flow density, CT, choroidal thickness. 


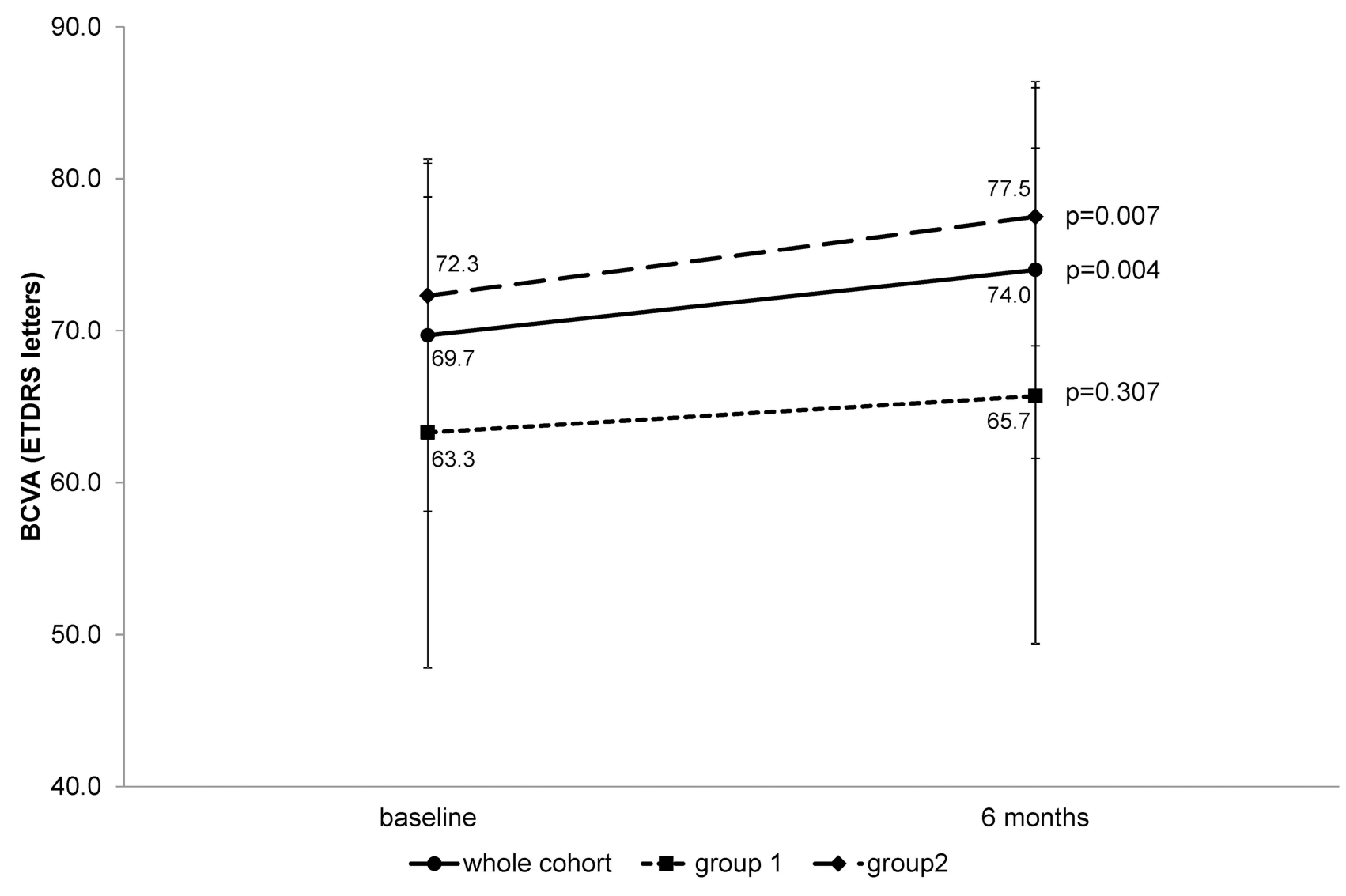

Figure I Change in BCVA from baseline to 6 months of follow-up.

therapy $(0.462, \mathrm{p}=0.023)$ and DME duration (0.484, $\mathrm{p}=0.017)$.

\section{BCVA and CFT Changes Over Time}

There was an improvement in BCVA both in the whole cohort and in group $2(+4.3$ ETDRS letters, $p=0.004$ and +5.2 ETDRS letters, $\mathrm{p}=0.007$, respectively), with no differences in group 1 (Figure 1). A significant decrease in CFT was observed in the whole cohort and in both groups (Figure 2).

\section{Choroidal Vascularity Changes Over Time}

Globally, the choroidal vascularity parameters CVI, CCD and $\mathrm{CT}$, did not change with ranibizumab treatment ( $>0.05$ ). Considering only group 2, there was a significant decrease in $\mathrm{CT}(\mathrm{p}=0.033)$ and a significant increase in $\operatorname{CCD}(\mathrm{p}=0.010) 6$ months after treatment, with no differences in CVI (Table 2).

\section{Correlations with Choroidal Vascularity at Month 6}

At month 6, CVI was moderately negatively correlated with body mass index (BMI) $-r=0.660, p=0.001$. The duration of treatment with insulin and previous treatments (macular LASER, PRP LASER, or anti-VEGF IV treatments) were not associated with choroidal vascularity parameters at week 24. Blood pressure did not show any influence in choroidal variables at week 24 .

\section{Prognostic Value of Baseline Choroidal Vascularity for Anatomical or Functional Response}

Despite no correlation with baseline BCVA, baseline CVI was positively correlated with final BCVA globally $(r=0.406$, $\mathrm{p}=0.029)$ and in group $2(r=0.604, \mathrm{p}=0.004)$. No other baseline choroidal parameters were correlated with final BCVA or final CFT. At 6 months, the same correlation was maintained between CVI and BCVA $(r=0.450, \mathrm{p}=0.041)$. 


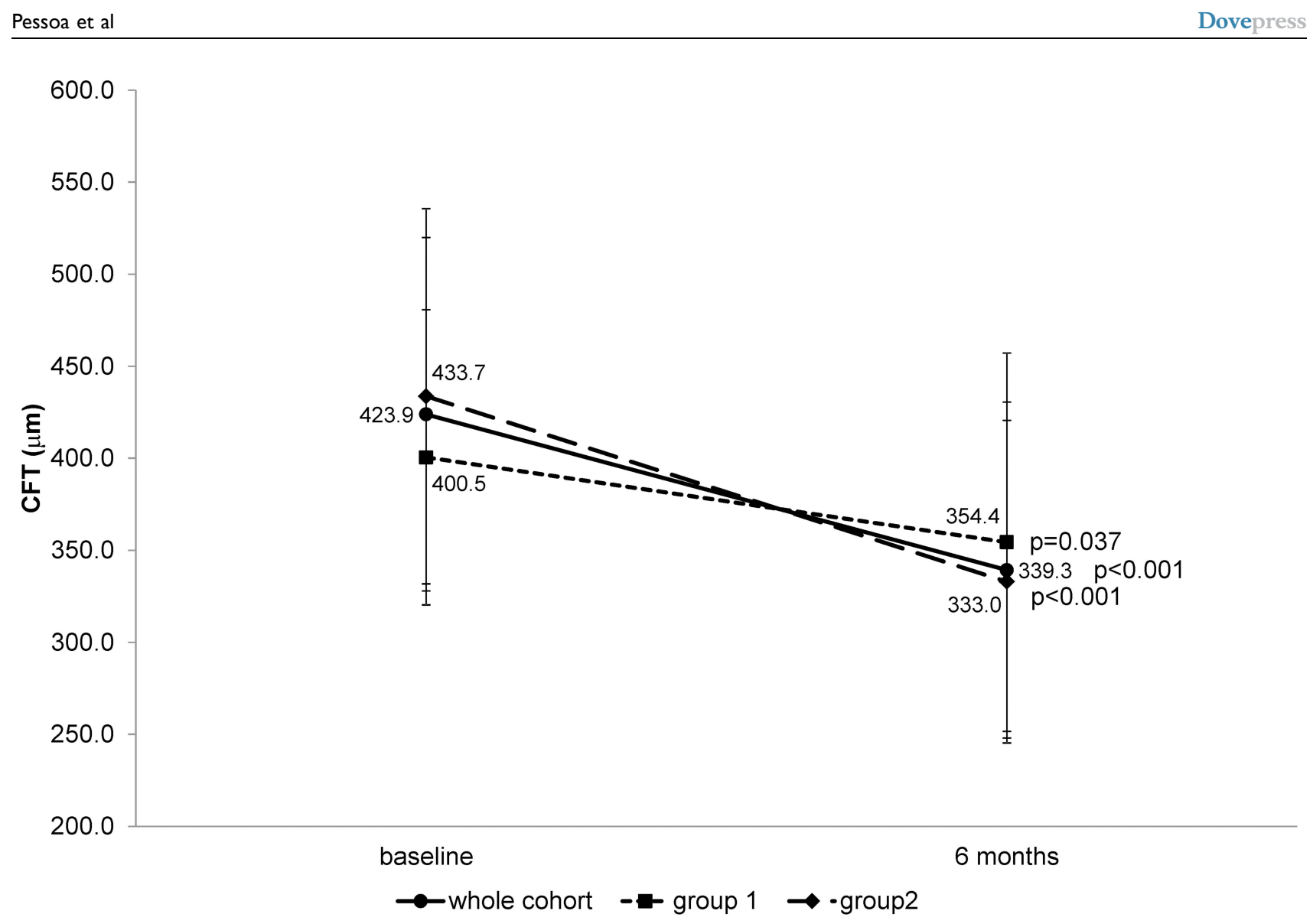

Figure 2 Change in CFT from baseline to 6 months of follow-up.

\section{Discussion}

There is growing evidence that choroidal vascularization, blood flow, and thickness are affected in diabetes and also influenced by intravitreal corticosteroid (CCT) and antiVEGF treatments. Nonetheless, their exact influence is not completely clarified. ${ }^{12-14}$ There are contradictory results regarding choroidal thickness in eyes with diabetic retinopathy, with or without DME, with different reports suggesting choroidal thickening, thinning, or no change. ${ }^{4,15-17}$ The retrospective and cross-sectional nature of most previous studies, the influence of different factors such as age, ${ }^{18}$ refractive error, ${ }^{19}$ different DR severity/ DME/PRP status, ${ }^{16,17}$ as well as the possible effect of previous anti-VEGF therapy, ${ }^{17}$ circadian cycle or even

Table 2 Changes in CVI, CCD, and CT, Globally and by Group

\begin{tabular}{|l|c|c|c|l|}
\hline & Group I [n=10] & Group 2 (n=24) & Total $(\mathbf{n = 3 4 )}$ & \\
\hline CT, $\mu \mathrm{m}$ & $233.7 \pm 73.7$ & $256.1 \pm 52.7$ & $250.0 \pm 58.7$ & Baseline \\
& $272.6 \pm 87.1$ & $245.5 \pm 55.9$ & $254.2 \pm 67.3$ & 6 -months \\
& $+38.9, \mathrm{p}=0.109$ & $-10.6, \mathbf{p}=\mathbf{0 . 0 4 2}$ & $+4.2, \mathrm{p}=0.508$ & Change, $p$-value \\
\hline CVI, \% & $63.3 \pm 3.5$ & $64.1 \pm 6.5$ & $63.9 \pm 5.8$ & Baseline \\
& $63.5 \pm 6.1$ & $66.2 \pm 5.6$ & $65.3 \pm 5.8$ & 6 -months \\
& $+0.2, \mathrm{p}=0.945$ & $+2.1, \mathrm{p}=0.111$ & $+1.4, \mathrm{p}=0.169$ & Change, $p$-value \\
\hline CCD, \% & $39.3 \pm 7.2$ & $37.3 \pm 11.6$ & $37.9 \pm 10.4$ & Baseline \\
& $39.3 \pm 11.3$ & $41.2 \pm 10.9$ & $40.6 \pm 10.9$ & 6 -months \\
& $0.0, \mathrm{p}=0.109$ & $+3.9, \mathbf{p}=\mathbf{0 . 0 4 2}$ & $+3.7, \mathrm{p}=0.100$ & Change, $p$-value \\
\hline
\end{tabular}

Notes: Significant P-values in bold. Values are shown as mean \pm standard deviation. P-values from Wilcoxon tests.

Abbreviations: group I, vitrectomized eyes; group 2, non-vitrectomized eyes; BCVA, best-corrected visual acuity; CFT, central foveal thickness; CT, choroidal thickness; $\mathrm{CVI}$, choroidal vascularity index; CCD, choriocapillaris flow density. 
other systemic vascular factors such as blood pressure ${ }^{20}$ may be among the reasons for these disparities.

Our study aimed to clarify the effect of intravitreal RBZ therapy in a PRN regimen for DME in choroidal vascular tissue, comparing vitrectomized with nonvitrectomized eyes, during 24 weeks of follow-up, an acceptable period to reach anatomical and functional stability or to establish the type of response to anti-VEGF. ${ }^{21}$

Regarding baseline demographics, the only difference between groups was DM duration. This was to be expected since vitreopathy with vitreoretinal interface pathological changes, and a more severe and refractory DR, are consequences of the cumulative effect of hyperglycemia, which increases the probability of the need for vitrectomy. ${ }^{22}$ There are evidences in the literature that in cases with ERM and indication for vitrectomy, CT and CVI are increased, with gradual reduction after surgery. ${ }^{23,24}$ In one study evaluating and comparing CVI with a control group, the decrease occurred until the thickness matched that of the normal fellow eye. The secondary inflammation resulting from mechanical traction was the explanation for the increased choroidal thickness by way of increased vascularization of the choroid. ${ }^{24}$ In line with this last study, our observation that baseline choroidal parameters were not significantly different between groups may reflect the fact that vitrectomy (with ILM peeling, performed in the majority of patients) by itself does not induce detectable choroidal changes in diabetic eyes.

In order to decrease the bias due to other important factors that could influence choroidal flow or thickness, all measurements were performed at the same time of the day, high myopic eyes were excluded, and the remaining baseline characteristics were included in the analysis. Age, BMI, BCVA, CFT, and previous treatments, such as LASER and anti-VEGF treatments, were not different between groups. The absence of correlation of any baseline characteristics with CT, CVI or CCD, reinforces the uniformity among patients included in our study.

The main clinical consequence of anti-VEGF, the firstline therapy for $\mathrm{DME},{ }^{25}$ is to induce DME regression that leads to the expected functional improvement. This was observed in our study for both groups, although with a statistical significance only for group 2, particularly regarding BCVA. We may speculate that the lower functional outcome in group 1 may be due to a faster clearance of drugs in vitrectomized eyes, leading to a final higher $\mathrm{CFT}$, which may have an impact on the final $\mathrm{BCVA},{ }^{26}$ on the ILM peeled feature, in $80 \%$ of these eyes, or on the longer DM duration. These last two factors are correlated with retina neurodegeneration, manifested by lower inner retina layers' thickness, which may negatively influence the functional outcome. ${ }^{27-29}$ The need for vitrectomy in a context of PDR (present in $80 \%$ of the eyes) may also be a sign of a more severe DR, in both neuronal and vascular degenerative components related to DR. Our results are also in line with the evidence that RBZ is an effective treatment for DME in vitrectomized eyes, although the functional and anatomical efficacy seems to be achieved slower, with the need for a higher number of injections at least during the first 12 months of treatment. ${ }^{30}$ Nevertheless, it has to be taken into account that our population is a cohort of diabetic patients that are well known for their clinical heterogeneity.

Analyzing the choroidal vascularity parameters, we found a decrease in $\mathrm{CT}$ and a significant increase in CCD only in group 2, even though choroidal parameters at baseline were similar in both groups. According to the literature, anti-VEGF treatment reduces choroidal thickness, probably due to the reduction of the choroidal vasculature permeability. ${ }^{14,15}$ On the other hand, we hypothesize that the reduced permeability of the large choroidal vessels reduces the interstitial fluid pressure and may favor blood flow in the choriocapillaris, as confirmed by the increase in CCD. Although there is robust evidence towards Diabetic Retinopathy Severity Scale (DRSS) score improvement and neovascularization regression in PDR cases, objective reperfusion in ischemic areas with anti-VEGF therapy, based on fluorescein angiography and OCT-A, yields more conflicting results. ${ }^{31}$ Subanalyses of the Phase 3 RISE and RIDE trials concluded that monthly injections of RBZ can slow, but not completely prevent, retinal capillary closure in patients with DME. ${ }^{10}$ In other recent studies, choriocapillaris and retinal capillary perfusion density remained unchanged after 3 or 12 months of treatment with anti-VEGF. ${ }^{32-34}$ Pongsachareonnont et al observed that all anti-VEGF medications reduce not only the number of microaneurysms but also the foveal avascular zone (FAZ) in the superficial and deep capillary plexus after one injection, with a positive correlation with visual improvement. ${ }^{35}$

Our results suggest that non-vitrectomized eyes may have a different response to anti-VEGF therapy in choroidal vascular anatomical readjustment, through the reduction of choroidal vasculature permeability, in addition to the renewal of the blood-retinal barriers. ${ }^{25}$ In the existing literature regarding choroidal thickness and retinal 
vascular density, vitrectomy is not an exclusion or grouping criteria. If that distinction had been done, then different conclusions could have emerged regarding macular perfusion across a great number of studies.

The absence of similar results for vitrectomized eyes, although it can be influenced by the small number of eyes included, may be due to 1) the less expressive effect of anti-VEGF in reducing not only retinal but also choroidal vasculature permeability, which is in line with a less dry macula observed in vitrectomized eyes and 2) the knowledge, already discussed above, that functional and anatomical efficacy also occur in vitrectomized eyes, although more slowly and depending on a high treatment burden, at least during the first year. ${ }^{30}$ A longer follow-up would help to corroborate these hypotheses. Otherwise, the potential higher neurovascular disease severity or intense PRP, usually added intraoperatively in the vitrectomized eyes included in this study, may also be possible explanations.

A novelty highlighted by our analyses was the observation that baseline CVI seems to play a role as a potential functional predictor of anti-VEGF response in DME, overall and in non-vitrectomized eyes.

In our study, and contradicting the results obtained by Rayess et $\mathrm{al}^{36}$ after three monthly injections of antiVEGF, CT and CCD did not show an additional prognostic value regarding anatomical or functional response after 12 and 24 weeks of treatment. A possible explanation for otherwise early outcomes by these authors may lay on their different inclusion criteria: only naïve eyes, without previous treatments related to diabetic retinopathy before the first IV anti-VEGF injection. An expected higher baseline sub-foveal choroidal thickness in those eyes may represent a subgroup with more preserved choriocapillaris and thus greater potential for significant improvements following anti-VEGF therapy. In our cohort, the majority of patients were not naïve. Particularly, PRP LASER therapy is associated with a significant reduction of the subfoveal choroidal thickness and subfoveal choroidal blood flow. ${ }^{17} \mathrm{~A}$ similar effect of anti-VEGF therapy on CT has already been reported, although the data is scarce in the long term, such as a treatment pause of 6 months, considered for inclusion in our study. ${ }^{12-14}$

Another expected result from our findings was the correlation between the number of injections needed to control DME with DME duration and insulin therapy, anticipating a more severe vascular damage and a higher treatment burden to stabilize the diabetic neurovascular disease.

The main limitation of this study is the reduced sample size, particularly in group 1 . The small number of vitrectomized eyes included in this study is a reflection of the relatively infrequent post-vitrectomy DME occurrence. ${ }^{37,38}$ The prospective evaluation of our cohort, the relatively uniform demographic characteristics (besides the expected differences associated with the need for vitrectomy), and our attempt to control for potential confounders are the strong points of our methodology.

Although we chose 24 weeks of follow-up as an acceptable and robust period to reach anatomical and functional stability, a longer prospective observation period might be needed to confirm our results. In addition, the known artifacts associated with OCT-A images and their analysis might lead to misinterpretations.

\section{Conclusions}

In conclusion, and in our population sample, CCD increased and CT decreased in non-vitrectomized eyes after 6 months of PRN treatment with IV RBZ. Moreover, CVI demonstrated to be a potential predictor for functional response to RZB therapy in DME. Choroid vascular status may help to understand different functional prognosis observed with anti-VEGF treatment, that classical retinal biomarkers cannot otherwise explain.

Future studies with larger samples should be carried out to further investigate and confirm our results with regards to the role of vitreous status in response to choroidal vascular tissue to anti-VEGF therapy.

\section{Abbreviations}

BCVA, best-corrected visual acuity; BMI, body mass index; $\mathrm{BP}$, blood pressure; $\mathrm{CA}$, choroidal area; $\mathrm{CCD}$, choriocapillaris flow density; CFT, central subfield foveal thickness; CT, choroidal thickness; CVI choroidal vascular index; DM, diabetes mellitus; DME, diabetic macular edema; DR, diabetic retinopathy; DRSS, diabetic retinopathy severity scale; ERM, epiretinal membrane; ETDRS, early treatment of diabetic retinopathy study; FAZ, foveal avascular zone; HbA1C, glycated hemoglobin; ILM, internal limiting membrane; IV, intravitreal injection; LA, luminal areas; OCT, optical coherence tomography; PDR, proliferative diabetic retinopathy; PRN, pro re nata; PRP, panretinal photocoagulation; RBZ, ranibizumab; SCS, suprachoroidal space; SD-OCT, spectral domain optical coherence tomography; VEGF, vascular endothelial growth factor. 


\section{Data Sharing Statement}

The datasets generated during and/or analyzed during the current study are available from the corresponding author on reasonable request.

\section{Ethics Approval and Informed Consent}

This study was conducted according to the tenets of the Declaration of Helsinki in its latest amendment (Brazil, 2013) and was approved by the ethics committee of CHUP [2017.093 (084-DEFI/082-CES)]. All patients signed an informed consent form. This study is registered at www. clinicaltrials.gov (NCT04387604).

\section{Author Contributions}

We declare that all authors have made a significant contribution to the work reported, whether in the conception, study design, execution, acquisition of data, or analysis and interpretation; have drafted or written, or critically reviewed the article; have agreed on the journal to which the article was submitted; reviewed and agreed on all versions of the article before submission, during revision, the final version accepted for publication, and any significant changes introduced at the proofing stage; and agree to take responsibility and be accountable for the contents of the article.

\section{Disclosure}

The authors declare no competing interests, either financial or non-financial.

\section{References}

1. Yau JW, Rogers SL, Kawasaki R, et al. Global prevalence and major risk factors of diabetic retinopathy. Diabetes Care. 2012;35 (3):556-564. doi:10.2337/dc11-1909

2. Bhagat N, Grigorian RA, Tutela A, Zarbin MA. Diabetic macular edema: pathogenesis and treatment. Surv Ophthalmol. 2009;54 (1):1-32. doi:10.1016/j.survophthal.2008.10.001

3. Hidayat AA, Fine BS. Diabetic choroidopathy. Light and electron microscopic observations of seven cases. Ophthalmology. 1985;92 (4):512-522. doi:10.1016/S0161-6420(85)34013-7

4. Querques G, Lattanzio R, Querques L, et al. Enhanced depth imaging optical coherence tomography in type 2 diabetes. Invest Ophthalmol Vis Sci. 2012;53(10):6017-6024. doi:10.1167/iovs.12-9692

5. Esmaeelpour M, Považay B, Hermann B, et al. Mapping choroidal and retinal thickness variation in type 2 diabetes using three-dimensional 1060-nm optical coherence tomography. Invest Ophthalmol Vis Sci. 2011;52(8):5311-5316. doi:10.1167/iovs.10-6875

6. Totan Y, Akyüz TK, Güler E, Güragaç FB. Evaluation of ocular pulse amplitude and choroidal thickness in diabetic macular edema. Eye. 2016;30(3):369-374. doi:10.1038/eye.2015.232

7. Maharaj AS, D'Amore PA. Roles for VEGF in the adult. Microvasc Res. 2007;74(2-3):100-113. doi:10.1016/j.mvr.2007.03.004
8. Saint-Geniez M, Maldonado AE, D'Amore PA. VEGF expression and receptor activation in the choroid during development and in the adult. Invest Ophthalmol Vis Sci. 2006;47(7):3135-3142. doi:10.1167/iovs.051229

9. Blaauwgeers HG, Holtkamp GM, Rutten H, et al. Polarized vascular endothelial growth factor secretion by human retinal pigment epithelium and localization of vascular endothelial growth factor receptors on the inner choriocapillaris. Evidence for a trophic paracrine relation. $\mathrm{Am}$ J Pathol. 1999;155(2):421-428. doi:10.1016/s0002-9440(10)65138-3

10. Campochiaro PA, Wykoff CC, Shapiro H, Rubio RG, Ehrlich JS. Neutralization of vascular endothelial growth factor slows progression of retinal nonperfusion in patients with diabetic macular edema. Ophthalmology. 2014;121(9):1783-1789. doi:10.1016/j.ophtha.201 4.03.021

11. Ghasemi Falavarjani K, Iafe NA, Hubschman JP, Tsui I, Sadda SR, Sarraf D. Optical Coherence tomography angiography analysis of the foveal avascular zone and macular vessel density after anti-VEGF therapy in eyes with diabetic macular edema and retinal vein occlusion. Invest Ophthalmol Vis Sci. 2017;58(1):30-34. doi:10.1167/iovs.16-20579

12. Okamoto M, Yamashita M, Ogata N. Effects of intravitreal injection of ranibizumab on choroidal structure and blood flow in eyes with diabetic macular edema. Graefe's Arch Clin Exp Ophthalmol. 2018;256(5):885-892. doi:10.1007/s00417-018-3939-3

13. Sonoda S, Sakamoto T, Yamashita T, et al. Effect of intravitreal triamcinolone acetonide or bevacizumab on choroidal thickness in eyes with diabetic macular edema. Invest Ophthalmol Vis Sci. 2014;55(6):3979-3985. doi:10.1167/iovs.14-14188

14. Laíns I, Figueira J, Santos AR, et al. Choroidal thickness in diabetic retinopathy: the influence of antiangiogenic therapy. Retina. 2014;34 (6):1199-1207. doi:10.1097/iae.0000000000000053

15. Yiu G, Manjunath V, Chiu SJ, Farsiu S, Mahmoud TH. Effect of anti-vascular endothelial growth factor therapy on choroidal thickness in diabetic macular edema. Am J Ophthalmol. 2014;158(4):745751 e2. doi:10.1016/j.ajo.2014.06.006

16. Okamoto M, Matsuura T, Ogata N. Effects of panretinal photocoagulation on choroidal thickness and choroidal blood flow in patients with severe nonproliferative diabetic retinopathy. Retina. 2016;36 (4):805-811. doi:10.1097/iae.0000000000000800

17. Kim JT, Lee DH, Joe SG, Kim JG, Yoon YH. Changes in choroidal thickness in relation to the severity of retinopathy and macular edema in type 2 diabetic patients. Invest Ophthalmol Vis Sci. 2013;54 (5):3378-3384. doi:10.1167/iovs.12-11503

18. Margolis R, Spaide RF. A pilot study of enhanced depth imaging optical coherence tomography of the choroid in normal eyes. $\mathrm{Am}$ $J$ Ophthalmol. 2009;147(5):811-815. doi:10.1016/j.ajo.2008.1 2.008

19. Fujiwara T, Imamura Y, Margolis R, Slakter JS, Spaide RF. Enhanced depth imaging optical coherence tomography of the choroid in highly myopic eyes. Am J Ophthalmol. 2009;148(3):445-450. doi:10.1016/j. ajo.2009.04.029

20. Usui S, Ikuno Y, Akiba M, et al. Circadian changes in subfoveal choroidal thickness and the relationship with circulatory factors in healthy subjects. Invest Ophthalmol Vis Sci. 2012;53(4):2300-2307. doi:10.1167/iovs.11-8383

21. Bressler SB, Ayala AR, Bressler NM, et al. Persistent macular thickening after ranibizumab treatment for diabetic macular edema with vision impairment. JAMA Ophthalmol. 2016;134(3):278-285. doi:10.1001/jamaophthalmol.2015.5346

22. Sebag J. Abnormalities of human vitreous structure in diabetes. Graefe's Arch Clin Exp Ophthalmol. 1993;231(5):257-260. doi:10.1007/bf00919101

23. Michalewska Z, Michalewski J, Ornafel-Sagan K, Nawrocki J. Swept-source optical coherence tomography correlations between retina and choroid before and after vitrectomy for epiretinal membranes. Am J Ophthalmol. 2016;165:100-107. doi:10.1016/j. ajo.2016.02.003 
24. Rizzo S, Savastano A, Finocchio L, Savastano MC, Khandelwal N, Agrawal R. Choroidal vascularity index changes after vitreomacular surgery. Acta Ophthalmol. 2018;96(8):e950-e955. doi:10.1111/ aos. 13776

25. Schmidt-Erfurth U, Garcia-Arumi J, Bandello F, et al. Guidelines for the management of diabetic macular edema by the European Society of Retina Specialists (EURETINA). Ophthalmologica $J$ Int D'ophtalmologie Int J Ophthalmol Zeitschrift Fur Augenheilkunde. 2017;237(4):185-222. doi:10.1159/000458539

26. Ahn SJ, Ahn J, Park S, et al. Intraocular pharmacokinetics of ranibizumab in vitrectomized versus nonvitrectomized eyes. Invest Ophthalmol Vis Sci. 2014;55(1):567-573. doi:10.1167/iovs.13-13054

27. Won JY, Kim M, Park YH. Postoperative changes in the retinal thickness and volume after vitrectomy for epiretinal membrane and internal limiting membrane peeling. Medicine. 2017;96(19):e6709. doi: $10.1097 / \mathrm{md} .0000000000006709$

28. Sivalingam A, Eagle RC Jr, Duker JS, et al. Visual prognosis correlated with the presence of internal-limiting membrane in histopathologic specimens obtained from epiretinal membrane surgery. Ophthalmology. 1990;97(11):1549-1552. doi:10.1016/s01616420(90)32378-3

29. Simó R, Stitt AW, Gardner TW. Neurodegeneration in diabetic retinopathy: does it really matter? Diabetologia. 2018;61(9):1902-1912. doi:10.1007/s00125-018-4692-1

30. Bressler SB, Melia M, Glassman AR, et al. Ranibizumab plus prompt or deferred laser for diabetic macular edema in eyes with vitrectomy prior to anti-vascular endothelial growth factor therapy. Retina. 2015;35(12):2516-2528. doi:10.1097/iae.0000000000000617

31. Elnahry AG, Abdel-Kader AA, Habib AE, Elnahry GA, Raafat KA, Elrakhawy K. Review on recent trials evaluating the effect of intravitreal injections of anti-VEGF agents on the macular perfusion of diabetic patients with diabetic macular edema. Rev Recent Clin Trials. 2020;15(3):188-198. doi:10.2174/157488711566620051 9073704

32. Conti FF, Song W, Rodrigues EB, Singh RP. Changes in retinal and choriocapillaris density in diabetic patients receiving anti-vascular endothelial growth factor treatment using optical coherence tomography angiography. Int $j$ retina Vitreous. 2019;5:41. doi:10.1186/ s40942-019-0192-9
33. Sorour OA, Sabrosa AS, Yasin Alibhai A, et al. Optical coherence tomography angiography analysis of macular vessel density before and after anti-VEGF therapy in eyes with diabetic retinopathy. Int Ophthalmol. 2019;39(10):2361-2371. doi:10.1007/s10792-01901076-x

34. Couturier A, Rey PA, Erginay A, et al. Widefield OCT-angiography and fluorescein angiography assessments of nonperfusion in diabetic retinopathy and edema treated with anti-vascular endothelial growth factor. Ophthalmology. 2019;126(12):1685-1694. doi:10.1016/j. ophtha.2019.06.022

35. Pongsachareonnont P, Charoenphol P, Hurst C, Somkijrungroj T. The effect of anti-vascular endothelial growth factor on retinal microvascular changes in diabetic macular edema using swept-source optical coherence tomography angiography. Clin Ophthalmol. 2020;14:3871-3880. doi:10.2147/opth.s270410

36. Rayess N, Rahimy E, Ying GS, et al. Baseline choroidal thickness as a predictor for response to anti-vascular endothelial growth factor therapy in diabetic macular edema. Am J Ophthalmol. 2015;159 (1):85-91e1-3. doi:10.1016/j.ajo.2014.09.033

37. Pessoa B, Dias DA, Baptista P, Coelho C, Beirão JNM, Meireles A. Vitrectomy outcomes in eyes with tractional diabetic macular edema. Ophthalmic Res. 2019;61(2):94-99. doi:10.1159/000489459

38. Antoszyk AN, Glassman AR, Beaulieu WT, et al. Effect of intravitreous aflibercept vs vitrectomy with panretinal photocoagulation on visual acuity in patients with vitreous hemorrhage from proliferative diabetic retinopathy: a randomized clinical trial. JAMA. 2020;324 (23):2383-2395. doi:10.1001/jama.2020.23027

39. Agrawal R, Gupta P, Tan KA, Cheung CM, Wong TY, Cheng CY. Choroidal vascularity index as a measure of vascular status of the choroid: measurements in healthy eyes from a population-based study. Sci Rep. 2016;6:21090. doi:10.1038/srep21090

40. Chu Z, Gregori G, Rosenfeld PJ, Wang RK. Quantification of choriocapillaris with optical coherence tomography angiography: a comparison study. Am J Ophthalmol. 2019;208:111-123. doi:10.1016/j.ajo.2019.07.003
Clinical Ophthalmology

\section{Publish your work in this journal}

Clinical Ophthalmology is an international, peer-reviewed journal covering all subspecialties within ophthalmology. Key topics include: Optometry; Visual science; Pharmacology and drug therapy in eye diseases; Basic Sciences; Primary and Secondary eye care; Patient Safety and Quality of Care Improvements. This journal is indexed on PubMed

\section{Dovepress}

Central and CAS, and is the official journal of The Society of Clinical Ophthalmology (SCO). The manuscript management system is completely online and includes a very quick and fair peer-review system, which is all easy to use. Visit http://www.dovepress.com/ testimonials.php to read real quotes from published authors. 